\title{
Establishing Acceptance Limits for Dissolution Performance Verification of USP Apparatus 1 and 2 Using USP Prednisone Tablets Reference Standard Lot Q0H398
}

\author{
Walter W. Hauck*, Chensheng Li, Erika S. Stippler, \\ and William E. Brown \\ U.S. Pharmacopeial Convention, Rockville, MD, USA
}

\begin{abstract}
The United States Pharmacopeial Convention has released Prednisone Tablets Reference Standard lot Q0H398 (Q0) to replace lot P11300 (P1) for performance verification testing of dissolution Apparatus 1 and 2 as mandated by United States Pharmacopeia General Chapter Dissolution $<711>$. This report presents the collaborative study data, development of the acceptance limits, and results from supporting work for this lot. The collaborative study involved 21 collaborators who provided data for both Apparatus 1 and Apparatus 2. The study also evaluated the stability of Lot Q0 based on the dissolution results using Apparatus 1 and 2. The authors determined the geometric mean and percent coefficient of variation acceptance limits for 6-, 7-, 8-, 12-, and 14-position dissolution testers. The results of the collaborative study were used to establish the acceptance limits for the PVT for Apparatus 1 and 2 using lot Q0. Analysts found similarity in the data as well as in the acceptance limits for lots $\mathrm{Q} 0$ and $\mathrm{P} 1$, and dissolution results for Lot $\mathrm{Q} 0$ with Apparatus 1 appear to be stable. As with Lot P1, the dissolution results for Lot Q0 with Apparatus 2 show some decrease over time, and the geometric mean acceptance limits were adjusted accordingly. Results yielded acceptance limits for Apparatus 1 and Apparatus 2 that are different from those for Lot P1—but not markedly so—and are suitable for continued use in the PVT.
\end{abstract}

\section{INTRODUCTION}

$\int$ issolution testing provides an important measure of the in vitro release of the drug substance(s) from a drug product and therefore is a valuable quality control tool and also has an important role in determining biowaivers. Performance verification testing (PVT) uses the dissolution results from a reference material as a means to compare the performance (trueness and precision) of the assembly set-up and other parameters with that statistically derived from a well-controlled collaborative study (1-6). The U.S. Pharmacopeia (USP) General Chapter Dissolution $<711>$ requires periodic performance testing of USP Apparatus 1 and 2 using USP Prednisone Tablets Reference Standards (RS). PVT with USP Prednisone Tablets RS, in conjunction with mechanical calibration, is a valuable tool in maintaining proper dissolution apparatus performance (7, 8). PVT helps ensure that appropriate measures are taken to control the following sources of significant variability in dissolution testing: dissolved gasses, vibration, vessel dimensions, analyst, and analytical procedure (9-12). The PVT also addresses sources of variability that are unknown (4).

Since 1978 the United States Pharmacopeial Convention (USP) has provided prednisone tablets for use in a PVT for dissolution Apparatus 1 and 2. The previous lot of USP Prednisone Tablets RS, P1, was released in March 2010.

${ }^{*}$ Corresponding author.
The next lot, Q0, was released in November 2011. The purpose of this article is to report the collaborative and supporting studies used to determine the PVT acceptance limits for Lot Q0 and thereby to establish the suitability of Lot Q0 for continuing use in the PVT.

\section{METHODS}

Lot Q0 is a newly manufactured batch of prednisone tablets that shares the same formulation and manufacturing process as the current lot P1. Changes from P1 included manufacturing site and packaging configuration. The tablets of lot Q0 were manufactured in December 2008 and were packaged in blisters in January 2009. The material used for the blister packaging of Q0 was Tekniflex VA300 (Tekni-Plex, Inc., Somerville, NJ, USA), which is a 250-mm polyvinyl chloride/75-mm polychlorotrifluoroethylene film with 0.008 -in paperbacked foil. Blister packaging was selected for this lot to improve the in-use stability of the reference material.

\section{Stability Study}

To evaluate the rate of change in the dissolution values, we evaluated samples of blister-packed Lot Q0 reference material stored in USP's warehouse, which is a secure, temperature-controlled environment $\left(20^{\circ} \mathrm{C}\right)$. This study was initiated in June 2010 as part of the planning for a collaborative study scheduled for early 2011. Samples have been 
pulled in three-month intervals from June 2010 through the present, and the dissolution behavior was investigated using both Apparatus 1 and Apparatus 2. To reduce potential instrument-induced variability, dissolution tests were conducted by one analyst on the same dissolution instruments using both USP Apparatus 1 and Apparatus 2.

\section{Collaborative Study}

We conducted a collaborative study starting on January 6,2011 , and ending on March 7, 2011, to determine the acceptance ranges for USP Prednisone Tablets RS Lot Q0 for the Apparatus 1 and 2 dissolution PVTs. Laboratories affiliated with USP $(n=2)$, the pharmaceutical industry $(n=10)$, instrument manufacturers $(n=6)$, and regulatory agencies $(n=3)$ from 11 countries participated in the collaborative study. We asked all collaborators to ensure that all tests at their laboratories were conducted by the same analyst on the same equipment, including the vessels and stirring elements. Each experiment consisted of six, seven, or eight vessels, depending on the configuration of the laboratory's dissolution equipment. Participants in the collaborative study were required to perform mechanical calibration and the PVT on their equipment six months before the collaborative study began. The authors sent samples to 24 collaborating laboratories for analysis, and 21 laboratories completed the study and submitted results. Every laboratory in this group performed tests for both apparatus on four blinded samples, two each from lots Q0 and P1.

\section{Statistical Analyses}

The statistical analysis followed the approach used previously for Lot P1 (2). For each apparatus, the statistical method was the restricted maximum likelihood (REML) estimation of a nested, random-effects model for the natural logarithm of percent dissolved. Specifically, experiment was nested within collaborator, and collaborator and experiment were random effects. Analyses were done in SAS 9.2 for Windows (SAS Institute Inc., Cary, NC, USA) using PROC MIXED. The default variance components covariance structure was used. This analysis estimated three variance components for Lot Q0-intercollaborator, interexperiment, and residual (within-experiment). Note that the residual variability included assay variability, any variability associated with position in the equipment, and tablet-totablet variability. All intermediate precision contributions were included within the intercollaborator variability.

The single-stage test acceptance limits for the geometric mean were determined from the collaborative study mean in the log scale, $\pm t$ standard deviations, where the standard deviation was the reproducibility standard deviation. For 2 sets of 6 tablets ( 12 tablets total), for example, this would be

$$
\exp \left(\bar{X} \pm t \sqrt{S_{C}^{2}+S_{E}^{2} / 2+S_{R}^{2} / 12}\right)
$$

where the mean, $\bar{X}$, and variances, $S^{2}$, were estimated from the collaborative study; the subscripts $C, E$, and $R$ stand for
interCollaborator, interExperiment, and Residual variance components, respectively; and $t=2.12$ based on the 16 degrees of freedom in the variance estimate for the mean. For the within-experiment variance in the log scale, the upper limit was found as $F^{*} S_{R}^{2}$, where $F$ is the upper $5 \%$ point of an $F$-distribution with numerator degrees of freedom of 10 for a 6-tablet apparatus. For 7-, 8-, 12-, and 14-position assemblies, the degrees of freedom were 12, 14, 11, and 13, respectively. The denominator degrees of freedom were 180 for Apparatus 1 and 181 for Apparatus 2, the number of degrees of freedom available for repeatability (withinexperiment). Using the $t$ and $F$ values instead of normal and chi-square values as for Lot P1 slightly widened the limits (2). The current choice was based on statistical prediction intervals and was adopted originally for PVT collaborative studies with small numbers of laboratories.

The limits for the two stages of the two-stage option were determined as previously reported $(2,3)$ with one exception: As reported earlier, the limits for the first stage of the two-stage test were determined as for the one-stage test but used $60 \%$ confidence rather than $95 \%$ confidence, making the intervals narrower. For the second stage of the two-stage test, the limits were determined to preserve the operating characteristics (probabilities of passing) from the one-stage test. The exception is that for the geometric mean limits at the first stage, we found that the interval could be widened from $60 \%$ confidence to $80 \%$ confidence without materially changing the operating characteristics of the test and while keeping the limits at the second stage the same as for the single-stage test. This change makes the first-stage limits for the geometric mean slightly wider than would otherwise have been the case.

All estimated variances and variance components, $S^{2}$, and the upper acceptance limits for the within-experiment variance in the natural log scale were transformed back to \%CVs in the original, percent-dissolved scale using the lognormal formula: $\% C V=100 * \sqrt{\exp \left(S^{2}\right)-1}$.

\section{RESULTS}

\section{Stability Studies}

Figure 1 shows the stability data to date for stored samples. Results for Apparatus 1 appear stable, but those for Apparatus 2 show a tendency to decrease over time. The authors evaluated the stability trend by fitting a regression model to the data. As with Lot P1 $(1,2)$, the linear model in the log scale fit the Lot Q0 data best, which indicates that the decrease in the dissolution values for Apparatus 2 follows an apparent first-order kinetics model. For both apparatus, there is no indication of any change in variability with time.

\section{Collaborative Study}

Results from 21 laboratories were received by the deadline and were used for statistical analyses.

The data were screened in three steps. First, the information received was screened for protocol violations. For screening step 1, collaborators were asked to provide 

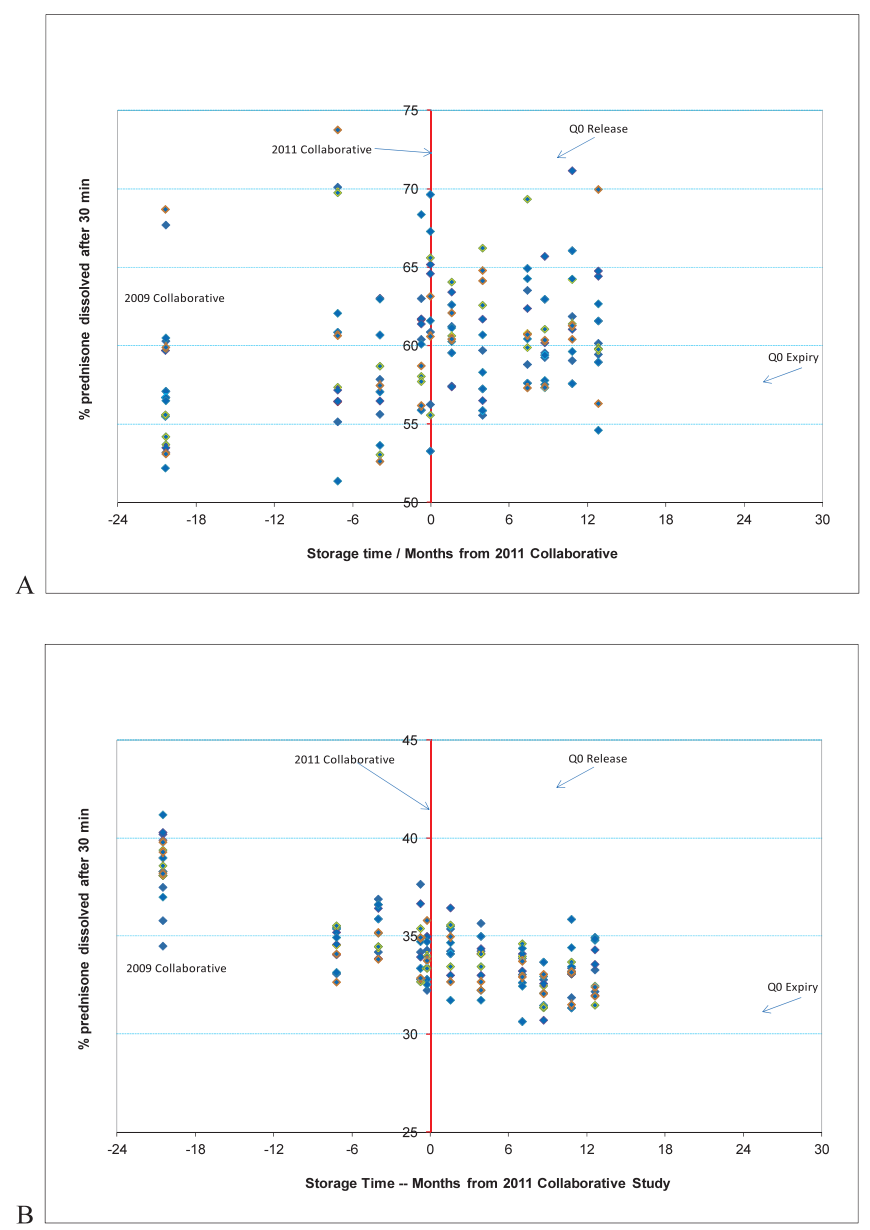

Figure 1.Q0 stability data for (A) Apparatus 1 and (B) Apparatus 2. Note that the vertical scales are in the center of the figures.

sufficient information to document that they followed the protocol as specified and to document that they had passed a PVT using Lot P1 within the previous six months. In summary, data from four collaborators was not used in the determination of the acceptance limits (see Table 1).

For screening step 2, we applied the exclusions of Table 1 and then analyzed the data for Lot Q0 using the nested model described above (to obtain variance estimates) then using $\bar{X}$ and $S$ control charts (in the natural log scale). These control charts identified individual experiments whose mean or variability was unusual relative to the other values in this study. For the Xbar charts, 3-sigma limits were applied using the reproducibility standard deviation (all estimated variance components). Three-sigma limits corresponded to 0.0027 probability in the two tails combined. For the $S$ control charts, the same two-tailed 0.0027 probability was used, but only the upper control limit was applied.

Figure 2 shows the control charts for Lot Q0. The control limits shown are for assemblies with 8 positions. The limits for 6- and 7-position assemblies are wider than those for 8 because they have fewer degrees of freedom. Excluding the laboratories listed in Table 1, only one experiment fell
Table 1. Laboratories Excluded from Analyses Because of Protocol Violations or Technical Failures

\begin{tabular}{cl}
\hline Laboratory & \multicolumn{1}{c}{ Reason } \\
\hline 9 & Protocol violation-non-USP degassing method \\
\hline 2 & $\begin{array}{l}\text { Provided two sets of data from different instruments; } \\
\text { used the data from the experiment listed first (note: this } \\
\text { is a technical failure, not a protocol violation) }\end{array}$ \\
\hline 14 & Technical error-absorptivity of standard solution high \\
\hline 17 & $\begin{array}{l}\text { Protocol violation-inadequate heating of medium for } \\
\text { degassing }\end{array}$ \\
\hline 21 & $\begin{array}{l}\text { No PVT in previous six months (mechanical calibration } \\
\text { only) }\end{array}$ \\
\hline
\end{tabular}

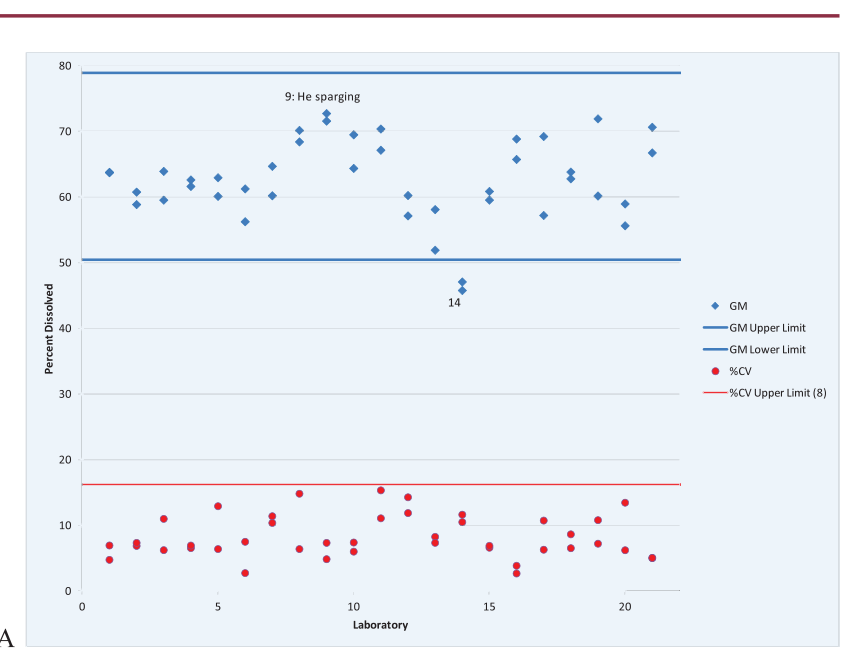

A

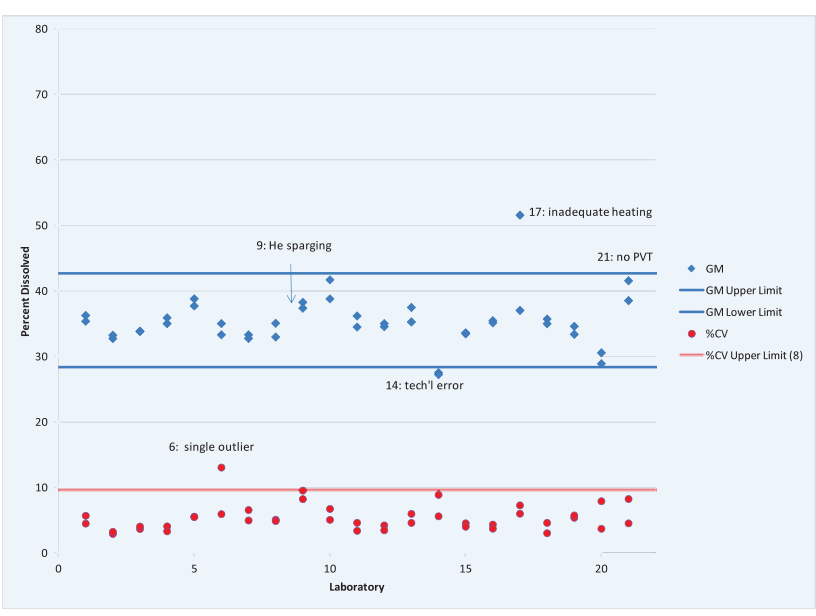

Figure 2. Control charts for (A) Apparatus 1 and (B) Apparatus 2. Horizontal lines are control limits for an instrument with eight positions. Limits for 6and 7-position instruments are slightly wider.

outside the control limits, namely laboratory 6,5 chart for Apparatus 2. When we examined the data, it was clear there was a single outlier (a value of 45.1 in the first experiment compared to a range of 32.2-35.9 for the remaining five values). This experiment was left in the analyses, and the individual value was considered at the next screening step. 
For screening step 3, the exclusions of Table 1 were applied, and the data were analyzed again using the nested model. These analyses were examined for individual tablet results that were outliers (i.e., unusual relative to the other values in the particular experiment). In the two analyses combined, there was one studentized residual (5.4) greater than 4.0 , a value that corresponded approximately to the $1 \%$ critical value for Grubb's test for outliers. On examination of Figure 3, we found that this candidate outlier was substantially separated from the remaining values of the experiment. This value, which was described in the immediately preceding paragraph, was dropped as an outlier.

Figure 3 shows all the data before any exclusions. In this figure, each symbol is one individual result, and a vertical set of six to eight symbols is one experiment. Collaborators listed in Table 1 are identified by code. Lot P1 and Lot Q0 data generally match up well within each laboratory. Differences between results by laboratory are more evident than the differences between the two lots.

For determination of the PVT acceptance limits for Lot Q0, we applied the exclusions of Table 1 and removed the
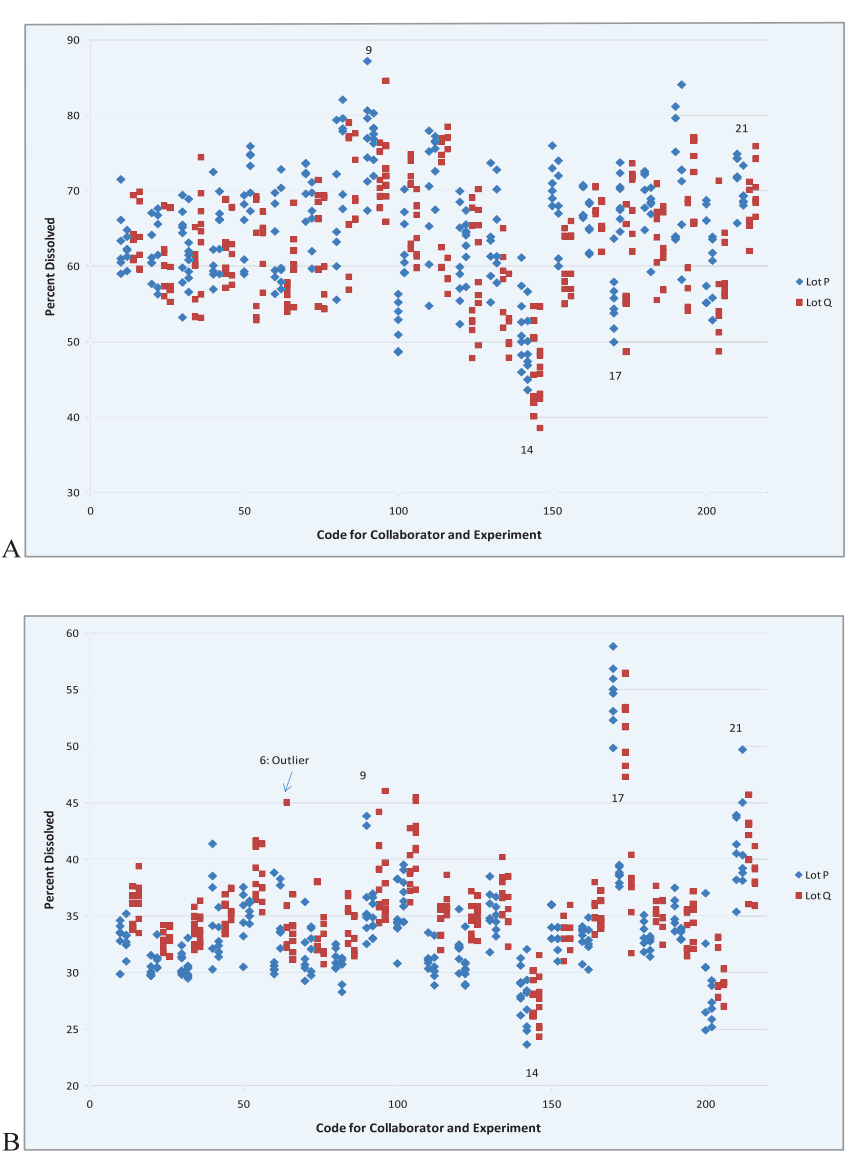

Figure 3. All data from the collaborative study (before any exclusions): (A) Apparatus 1 and (B) Apparatus 2. Laboratories marked are those listed in Table 1 that had protocol violations or technical failures. The outlier marked for Apparatus 2 is an unusual value relative to the others in that experiment and is discussed in the text. outlier. Table 2 shows the estimated geometric means and variance components (as \%CVs).

Because of the time trend (Figure 1B) in values for Apparatus 2, preliminary acceptance limits were adjusted following the procedure used for Lot P1 (Figure 4). (Note that Figure 4 shows only the stability data available before $\mathrm{Q} 0$ release and thus the only data available for determination of the trend.) The middle curve is the fitted regression (solid portion of curve) and the extrapolations (dashed curves) back to 2009 and forward to the Lot Q0 expiry date. One set of stability data was not used in the regression because it was much lower than trend, and no cause could be found. The slope corresponded to an approximate drop in potency of $5 \%$ relative to the percent dissolved (about $1.5 \%$ absolute/year). It is interesting, though not essential to the extrapolation forward in time, that the fitted curve does extrapolate back to data obtained in 2009.

Table 3 shows the new geometric mean and \%CV limits for both the one- and two-stage tests. For comparison, the single-stage PVT limits for Lot P1 also are shown. For Apparatus 2, the lower limits for the geometric mean were obtained by extrapolating preliminary lower limits to the Q0 expiry date, and the upper limits were obtained by extrapolating preliminary upper limits to the release date. These extrapolations are shown as two pairs of curves in

Table 2. Summary Mean and Variability Results for Lot QO

\begin{tabular}{|c|c|c|}
\hline & Apparatus 1 & Apparatus 2 \\
\hline Geometric Mean & 65 & 35 \\
\hline \multicolumn{3}{|l|}{$\%$ Coefficient of Variation } \\
\hline Between Collaborator & $4.8 \%$ & $6.3 \%$ \\
\hline Between Experiment & $4.7 \%$ & $1.8 \%$ \\
\hline $\begin{array}{l}\text { Within Experiment } \\
\text { (Residual) }\end{array}$ & $8.0 \%$ & $4.8 \%$ \\
\hline
\end{tabular}

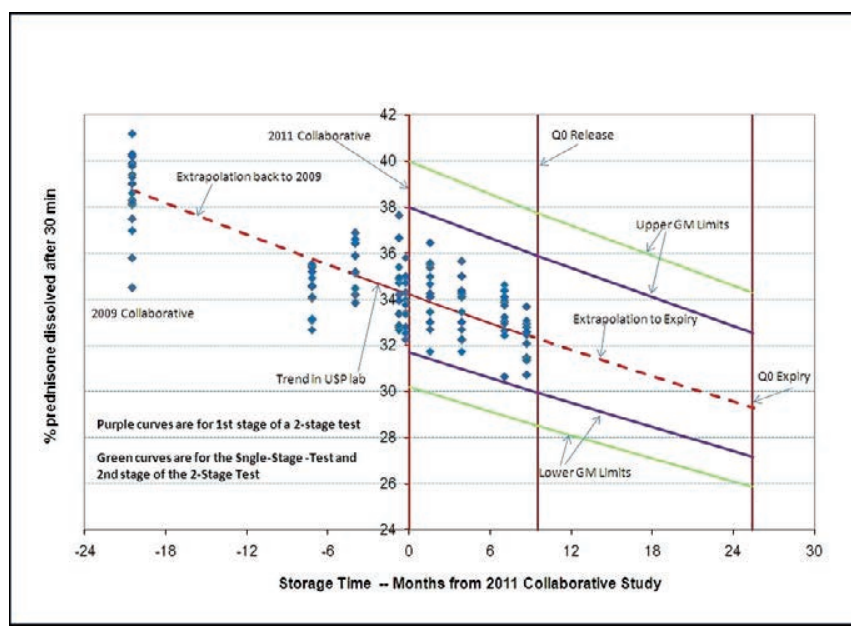

GM: geometric mean.

Figure 4. Time trend for Apparatus 2, Lot Q0. Note that the vertical axis scale is in the center of the figure. 
Table 3. Lot Q0 Geometric Mean/\% Coefficient of Variation (\%CV) Acceptance Limits

\begin{tabular}{|c|c|c|c|c|c|c|c|c|c|}
\hline & \multirow{3}{*}{ Positions } & \multicolumn{4}{|c|}{ Apparatus 1} & \multicolumn{4}{|c|}{ Apparatus 2} \\
\hline & & \multirow{2}{*}{$\frac{\text { Lot } \mathbf{P 1}}{\text { Single Stage }}$} & \multicolumn{3}{|c|}{ Lot Q0 } & \multirow{2}{*}{$\frac{\text { Lot P1 }}{\text { Single Stage }}$} & \multicolumn{3}{|c|}{ Lot Q0 } \\
\hline & & & Single Stage & $\begin{array}{l}1^{\text {st }} \text { Stage of } \\
\text { Two Stages }\end{array}$ & $\begin{array}{l}2^{\text {nd }} \text { Stage of } \\
\text { Two Stages }\end{array}$ & & Single Stage & $\begin{array}{l}1^{\text {st }} \text { Stage of } \\
\text { Two Stages }\end{array}$ & $\begin{array}{l}2^{\text {nd }} \text { Stage of } \\
\text { Two Stages }\end{array}$ \\
\hline Geometric Mean & 6,7, or 8 & $(56,75)$ & $(54,72)$ & $(57,69)$ & $(54,72)$ & $(25,41)$ & $(26,38)$ & $(27,36)$ & $(26,38)$ \\
\hline Limits & 12 or 14 & $(56,75)$ & $(54,72)$ & & & $(25,41)$ & $(26,38)$ & & \\
\hline \multirow{5}{*}{$\% C V$ Limits } & 6 & $10 \%$ & $12 \%$ & $9.2 \%$ & $12 \%$ & $6.8 \%$ & $6.7 \%$ & $4.9 \%$ & $6.5 \%$ \\
\hline & 7 & $10 \%$ & $12 \%$ & $9.2 \%$ & $12 \%$ & $6.7 \%$ & $6.5 \%$ & $4.9 \%$ & $6.3 \%$ \\
\hline & 8 & $10 \%$ & $12 \%$ & $9.3 \%$ & $12 \%$ & $6.5 \%$ & $6.4 \%$ & $5.0 \%$ & $6.3 \%$ \\
\hline & 12 & $10 \%$ & $12 \%$ & & & $6.7 \%$ & $6.6 \%$ & & \\
\hline & 14 & $10 \%$ & $12 \%$ & & & $6.6 \%$ & $6.5 \%$ & & \\
\hline
\end{tabular}

Figure 4. With these choices of limits, the laboratory using prednisone tablets for PVT is protected against any trend except a steeper drop in percent dissolved. From Figure 1B there is a suggestion that the trend is flattening. This will be analyzed as part of the determination of acceptance limits for the release of the next lot of this reference standard in 2012, which is a continuation lot of Q0. There is also an indication in Figure $1 \mathrm{~A}$ of an increasing trend for Apparatus 1. This will also be evaluated for the continuation lot.

\section{DISCUSSION}

In this paper, we have provided the data underlying the PVT acceptance limits for Prednisone Tablets RS, Lot Q0. Because of the similarities in formulation, manufacturing process, and dissolution behavior of lots Q0 and P1, Q0 tablets are expected to be as sensitive to the operational parameters as $\mathrm{P} 1(2,5,6,9-11)$. We see the similarity of the two Lots in Figure 3, where the data are similar, and in Table 3, where we see that the acceptance limits are similar. As a result, we conclude that analysts can confidently use USP Prednisone Tablets Reference Standard Lot Q0 for their PVTs of USP Apparatus 1 and 2.

\section{ACKNOWLEDGMENT}

The authors thank Stefan Schuber, Ph.D., M.S., ELS, director, scientific reports at USP, for editorial assistance.

\section{REFERENCES}

1. Deng, G.; Ashley, A. J.; Brown, W. E.; Eaton, J. W.; Hauck, W. W.; Kikwai, L. C.; Liddell, M. R.; Manning, R. G.;

Munoz, J. M.; Nithyanandan, P.; Glasgow, M. J.; Stippler, E.; Wahab, S. Z.; Williams R. L. The USP Performance Verification Test, Part I: USP Lot P Prednisone Tablets: quality attributes and experimental variables contributing to dissolution variance. Pharm. Res. 2008, 25 (5), 1100-1109.

2. Glasgow, M.; Dressman, S.; Brown, W. E.; Foster, T.; Schuber, S.; Manning, R. G.; Wahab, S. Z.; Williams, R. L.; Hauck, W. W. The USP Performance Verification Test, Part II: collaborative study of USP's Lot P Prednisone Tablets. Pharm. Res. 2008, 25 (5), 1110-1115.

3. Hauck, W. W.; DeStefano, A. J.; Brown, W. E.; Stippler, E. S.; Abernethy, D. R.; Koch, W. F.; Williams, R. L. Change in Criteria for USP Dissolution Performance Verification Tests. AAPS PharmSciTech 2009, 10 (1), 21-26.

4. Hauck, W. W.; Abernethy, D. R.; Williams, R. L. Metrologic approaches to setting acceptance criteria: Unacceptable and unusual characteristics. J. Pharm. Biomed. Anal. 2008, 48 (3), 1042-1045.

5. Nithyanandan, P.; Hauck, W. W.; Munoz, J.; Deng, G.; Brown, W.; Manning, R. G.; Wahab, S. Dissolution Variability: Comparison of Commercial Dosage Forms with US Pharmacopeia Lot P Prednisone Reference Standard Tablets-A Technical Note. AAPS PharmSciTech 2008, 9 (1), 238-242.

6. DeStefano, A. J.; Hauck, W. W.; Stippler, E. S.; Brown, W. E.; Li, C.; Huang, G. G.; Jones, B. J.; O'Hool, K.; Koch, W. F.; Williams, R. L. Establishing New Acceptance Limits for Dissolution Performance Verification of USPC Apparatus 1 and 2 Using USPC Prednisone Tablets Reference Standard. Pharm. Res. 2011, 28 (3), 505-516.

7. Manning, R. G.; Wahab, S. Z.; Brown, W. E.; Hauck, W. W.; Schuber, S.; Williams, R. L. Dissolution Testing and Metrological Measurement of Quality for Solid Oral Dosage Forms. Pharm. Technol. 2007, 31 (5), 68-74.

8. Foster, T. S.; Abernethy, D. R.; Koch, W. F.; Hauck, W. W. Inside USP: Metrology and USP Dissolution. Pharm. Technol. 2008, 32 (3), 86-90.

9. Nithyanandan, P.; Deng, G.; Brown, W.; Manning, R.; Wahab, S. Evaluation of the Sensitivity of USP Prednisone Tablets to Dissolved Gas in the Dissolution Medium Using USP Apparatus 2. Dissolution Technol. 2006, 13 (3), 15-18.

10. Eaton, J.; Deng, G.; Hauck, W. W.; Brown, W. E.; Manning, R. G.; Wahab, S. Perturbation Study of Dissolution Apparatus Variables-A Design of Experiment Approach. Dissolution Technol. 2007, 14 (1), 20-26.

11. Liddell, M. R.; Deng, G.; Hauck, W. W.; Brown, W. E.; Wahab, S. Z.; Manning, R. G. Evaluation of Glass Dissolution Vessel Dimensions and Irregularities. Dissolution Technol. 2007, 14 (2), 28-33.

12. Liddell, M.; Deng, G.; Hauck, W. W. Dissolution Testing Variability: Effect of Using Vessels from Different Commercial Sources. Am. Pharm. Rev. 2007, 10 (6), 122-128. 